\title{
The initial conditions for stellar protocluster formation
}

\section{The Herschel counterparts of the Spitzer Dark Cloud catalogue ${ }^{\star}$}

\author{
N. Peretto ${ }^{1}$, C. Lenfestey ${ }^{2}$, G. A. Fuller ${ }^{2}$, A. Traficante ${ }^{2}$, S. Molinari ${ }^{3}$, M. A. Thompson ${ }^{4}$, and D. Ward-Thompson ${ }^{5}$ \\ 1 School of Physics \& Astronomy, Cardiff University, Queen's building, The parade, Cardiff, CF24 3AA, UK \\ e-mail: nicolas.peretto@astro.cf.ac.uk \\ 2 Jodrell Bank Centre for Astrophysics, Alan Turing building, School of Physics \& Astronomy, The University of Manchester, \\ Oxford Road, Manchester M13 9PL, UK \\ 3 IAPS-Istituto di Astrofisica e Planetologia Spaziali, via Fosso del Cavaliere 100, 00133 Roma, Italy \\ 4 Centre for Astrophysics Research, University of Hertfordshire, College Lane, Hatfield, Herts AL10 9AB, UK \\ 5 Jeremiah Horrocks Institute, University of Central Lancashire, Preston, Lancashire PR1 2HE, UK
}

Received 27 July 2015 / Accepted 5 February 2016

\begin{abstract}
Context. Galactic plane surveys of pristine molecular clouds are key for establishing a Galactic-scale view of star formation. For this reason, an unbiased sample of infrared dark clouds in the $10^{\circ}<|l|<65^{\circ},|b|<1^{\circ}$ region of the Galactic plane was built using Spitzer $8 \mu \mathrm{m}$ extinction. However, intrinsic fluctuations in the mid-infrared background can be misinterpreted as foreground clouds.

Aims. The main goal of this study is to disentangle real clouds in the Spitzer Dark Cloud (SDC) catalogue from artefacts due to fluctuations in the mid-infrared background.

Methods. We constructed $\mathrm{H}_{2}$ column density maps at $\sim 18^{\prime \prime}$ resolution using the $160 \mu \mathrm{m}$ and $250 \mu \mathrm{m}$ data from the Herschel Galactic plane survey Hi-GAL. We also developed an automated detection scheme that confirms the existence of a SDC through its association with a peak on these Herschel column density maps. Detection simulations, along with visual inspection of a small sub-sample of SDCs, have been performed to get more insight into the limitations of our automated identification scheme.

Results. Our analysis shows that $76( \pm 19) \%$ of the catalogued SDCs are real. This fraction drops to $55( \pm 12) \%$ for clouds with angular diameters larger than $\sim 1$ arcmin. The contamination of the PF09 catalogue by large spurious sources reflects the large uncertainties associated to the construction of the $8 \mu \mathrm{m}$ background emission, a key stage in identiying SDCs. A comparison of the Herschel confirmed SDC sample with the BGPS and ATLASGAL samples shows that SDCs probe a unique range of cloud properties, reaching down to more compact and lower column density clouds than any of these two (sub-)millimetre Galactic plane surveys.

Conclusions. Even though about half of the large SDCs are spurious sources, the vast majority of the catalogued SDCs do have a Herschel counterpart. The Herschel-confirmed sample of SDCs offers a unique opportunity to study the earliest stages of both lowand high-mass star formation across the Galaxy.
\end{abstract}

Key words. catalogs - stars: formation - ISM: clouds - dust, extinction

\section{Introduction}

Only in recent years have technological breakthroughs made farinfrared/sub-millimetre Galactic plane surveys at sub-arcminute resolution possible (Schuller et al. 2009; Molinari et al. 2010; Aguirre et al. 2011). These surveys have, for the first time, the sensitivity and resolution to probe the individual dust clumps in which stars form, providing a high-resolution view of the star formation process on a Galactic scale. In particular, a complete understanding of the origin and distribution of stellar masses is only possible with large surveys of clumps that sample the full range of different physical properties and in which the initial conditions for star formation are still imprinted. In this context, performing a Galactic plane survey of infrared dark clouds (IRDCs) is essential.

IRDCs were first observed in 1996 by Perault et al. using ISOCAM at $15 \mu \mathrm{m}$ as absorption features against the infrared background of the Galactic plane. Since then, follow-up

* Full Table 1 is only available at the CDS via anonymous ftp to cdsarc.u-strasbg. fr (130.79.128.5) or via

http://cdsarc.u-strasbg.fr/viz-bin/qcat?]/A+A/590/A72 observations have shown that these sources are cold, dense molecular clouds and potential mass reservoirs for future generations of stars (e.g. Teyssier et al. 2002; Simon et al. 2006; Rathborne et al. 2006; Ragan et al. 2009). Their darkness in the mid-infrared domain ensures that these sources represent early stages in dense cloud evolution and, as such, IRDCs might contain the initial conditions of star formation. Peretto \& Fuller (2009, Paper I, hereafter PF09) constructed a catalogue of over 11000 Spitzer dark clouds (SDCs) using the $8 \mu \mathrm{m}$ GLIMPSE Galactic plane survey (Churchwell et al. 2009) covering the $10^{\circ}<|l|<65^{\circ},|b|<1^{\circ}$ region (see Fig. 1 for an example of a SDC). In PF09, $4^{\prime \prime}$ angular resolution column density maps were constructed from the $8 \mu \mathrm{m}$ extinction for all SDCs. This database has been used since for follow-up observations of specific clouds (Peretto et al. 2010, 2013, 2014), but also to tackle Galactic-scale problematics, such as the mass distribution of IRDCs and their sub-structures (Peretto \& Fuller 2010), the existence of column density thresholds for the formation of massive stars (Kauffmann \& Pillai 2010), or the characterisation of massive dense clumps (Traficante et al. 2015). This type of global study is the main reason behind building the PF09 catalogue in 

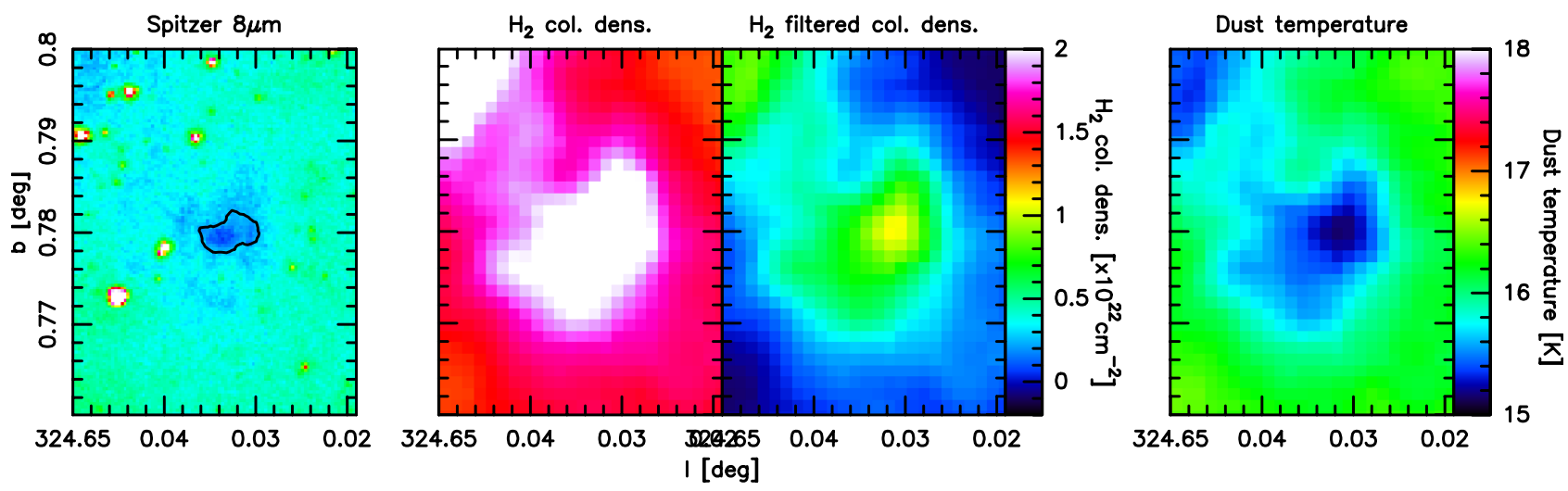

Fig. 1. Images of SDC324.633+0.779. From left to right: Spitzer $8 \mu \mathrm{m}$ image with the $\tau_{8} \mu \mathrm{m}=0.35$ opacity contour showing the boundary of the SDC as defined in PF09; Herschel $\mathrm{H}_{2}$ column density map with background; Herschel background filtered $\mathrm{H}_{2}$ column density map; Herschel dust temperature map.

the first place. However, the PF09 catalogue is contaminated by spurious clouds since any significant dip in the $8 \mu \mathrm{m}$ emission of the Galactic plane on angular scales lower than $\sim 5^{\prime}$ is considered to be the result of the extinction by a cloud, while it could simply be due to the intrinsic variation of the Galactic plane emission. Disentangling between real and spurious SDCs is therefore crucial for any Galactic-scale study that makes use of the PF09 catalogue.

Wilcock et al. (2012) looked at the $300^{\circ}<l<330^{\circ}$ region of the Galactic plane using Herschel Hi-GAL data and visually estimated that only $38 \%$ of the dark clouds from the PF09 catalogue were bright at $250 \mu \mathrm{m}$. Taken at face value, this suggests that most of the catalogued SDCs are artefacts, with only a minority of them real, casting doubt on any global-scale study that is based on the entire PF09 catalogue. However, a more rigorous approach to cloud identification is to systematically assess which SDCs are associated with peaks in the $\mathrm{H}_{2}$ column density determined from Hi-GAL data. This is the main objective of the present study.

In this paper, we present an analysis of the Herschel counterparts of all SDCs from the PF09 catalogue based on $\mathrm{H}_{2}$ column density images. Section 2 presents the observations. Section 3 describes how Herschel column density maps are constructed. Section 4 explains the identification scheme and its limitations. Section 5 presents a comparison between the automated and visual detection fractions of SDCs. In Sect. 6 we investigate the percentage of real SDCs that are detected in recent (sub-)millimetre Galactic plane surveys. Finally, summary and conclusions are in Sect. 7.

\section{Herschel data}

To confirm the nature of the SDCs, we use far-infrared dust emission data taken with the Herschel Space Observatory (Pilbratt et al. 2010). The two onboard photometry instruments, PACS (Poglitsch et al. 2010) and SPIRE (Griffin et al. 2010), allow the simultaneous observation of the dust emission at five wavelengths in the range $70-500 \mu \mathrm{m}$. The Hi-GAL open-time key project (Molinari et al. 2010) has observed the entire Galactic plane for a Galactic latitude $|b|<1^{\circ}$ at wavelengths of 70 , $160,250,350$, and $500 \mu \mathrm{m}$, offering a unique opportunity to study the dust emission properties of SDCs.

The Hi-GAL data were reduced, as described in Traficante et al. (2011), using HIPE (Ott 2010) for calibration and deglitching (SPIRE only), routines especially developed for Hi-GAL data reduction (drift removal, deglitching), and the ROMAGAL map-making algorithm. Post-processing on the maps was applied to help with image artefact removal (Piazzo et al. 2015). In this paper, we make use of only the PACS $160 \mu \mathrm{m}$ and SPIRE $250 \mu \mathrm{m}$ data with a nominal angular resolution of $\theta_{160}=12^{\prime \prime}$ and $\theta_{250}=18^{\prime \prime}$, respectively.

In addition, zero-flux levels for every Hi-GAL field have been recovered by correlating Herschel data with Planck and IRAS data (Bernard et al. 2010).

\section{Column density from Herschel}

The main goal of this study is to disentangle real from spurious SDCs in the PF09 catalogue. We believe that Herschel column density maps are probably the most suitable data to do so. In this section, we discuss the construction and reliability of our Herschel column density maps.

\subsection{Building $18^{\prime \prime}$ Herschel column density maps from the $160 \mu \mathrm{m} / 250 \mu \mathrm{m}$ colour}

A difference in angular resolution is a major issue when crosscorrelating two samples of sources. For this reason, it is essential here that we construct the highest possible angular resolution column density maps using the Herschel data. The typical way to construct Herschel column density maps is to perform pixel-bypixel SED fitting using Herschel data at four or five wavelengths (e.g. Peretto et al. 2010; Battersby et al. 2011). While this is the most reliable way of constructing such maps, it requires the data to be smoothed to the resolution at the longest wavelength, i.e. $\sim 36^{\prime \prime}$ at $500 \mu \mathrm{m}$. For the purpose of confirming whether a SDC corresponds to a column density peak, a simpler, faster analysis can be used, one that also produces higher angular resolution column density maps.

Here we use the ratio of the Hi-GAL $160 \mu \mathrm{m}$ over $250 \mu \mathrm{m}$ images as a temperature tracer, and use the derived temperature to estimate the column density from the $250 \mu \mathrm{m}$ data. The $160 \mu \mathrm{m}$-to- $250 \mu \mathrm{m}$ flux ratio, $R_{160 / 250}$, can be written as

$R_{160 / 250}=\frac{S_{160}}{S_{250}}=\frac{B_{v_{160}}\left(T_{\mathrm{d}}\right)}{B_{v_{250}}\left(T_{\mathrm{d}}\right)}\left(\frac{250}{160}\right)^{\beta}$

where $S_{\lambda}$ is the flux density at the wavelength $\lambda, B_{v}$ is the Planck function, $T_{\mathrm{d}}$ the dust temperature, and $\beta$ the spectral index of the specific dust opacity law set to 2 (Hildebrand 1983). As shown in Fig. 2, $R_{160 / 250}$ is a monotonic function of the dust temperature, so it can be used to estimate the dust temperature. In the 10-20 K temperature range, which is typical of IRDCs (Peretto et al. 2010), this ratio varies by a factor of $\sim 5$. In practice, because the $160 \mu \mathrm{m}$ and $250 \mu \mathrm{m}$ images originally have 


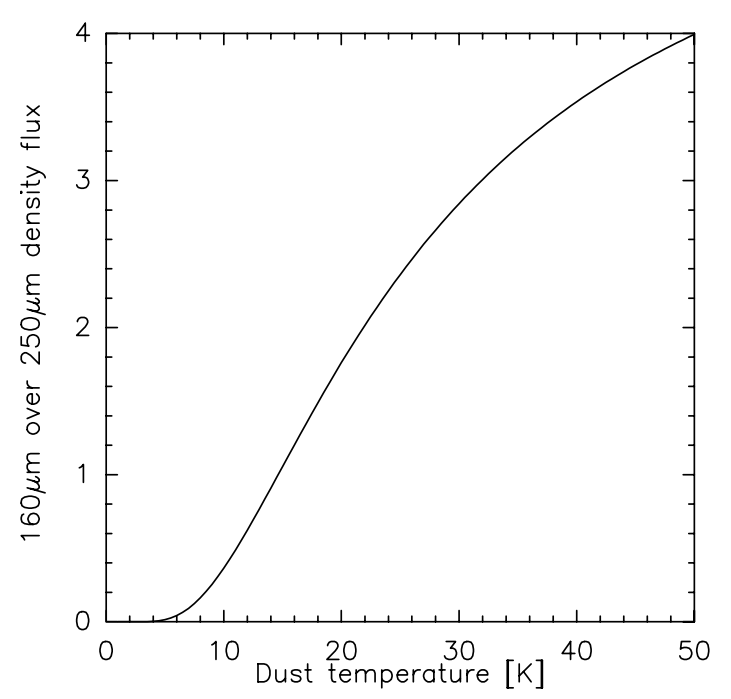

Fig. 2. Variation in $R_{160 / 250}$, the ratio between $160 \mu \mathrm{m}$ to $250 \mu \mathrm{m}$ flux density as a function of dust temperature (see Eq. (1)). A value of 2 was adopted for the spectral index of the dust opacity law (i.e. $\beta$ ).

different pixels sizes and projection centres, we regridded the $160 \mu \mathrm{m}$ images to match the $250 \mu \mathrm{m}$ image astrometry. We then convolved the $160 \mu \mathrm{m}$ images to the $250 \mu \mathrm{m}$ image resolution using a Gaussian kernel of FWHM $\theta_{\mathrm{ker}}=\sqrt{\theta_{250}^{2}-\theta_{160}^{2}}=13.4^{\prime \prime}$. We used the resulting convolved $160 \mu \mathrm{m}$ image with the original $250 \mu \mathrm{m}$ image to compute the $R_{160 / 250}$ ratio maps. We then converted the Hi-GAL $R_{160 / 250}$ maps into a temperature map. The signal-to-noise ratio in Hi-GAL maps is very high with a minimum value of 10 at 160 and $250 \mu \mathrm{m}$ for the faintest regions of the Galactic plane covered by Herschel (Molinari et al. 2016). This means that for the vast majority of the SDCs studied here, the uncertainty on $R_{160 / 250}$ is only a few percent, which translates into a temperature uncertainty of a few tenths of a Kelvin. To calculate the column density map, we then combine this temperature map with the Hi-GAL $250 \mu \mathrm{m}$ image, to derive the column density through the equation:

$N_{\mathrm{H}_{2}}=S_{250} /\left[B_{v_{250}}\left(T_{\mathrm{d}}\right) \kappa_{250} \mu \mathrm{m}_{\mathrm{H}}\right]$

where $\kappa_{250}=0.12 \mathrm{~cm}^{2} \mathrm{~g}^{-1}$ (Ossenkopf \& Henning 1994) is the specific dust opacity at $250 \mu \mathrm{m}$ (that already includes a dust-togas mass ratio of $1 \%), \mu=2.33$ is average molecular weight, and $m_{\mathrm{H}}$ the atomic mass of hydrogen. The simplicity of this method allows rapid construction of relatively high (i.e. $18^{\prime \prime}$ ) angular resolution column density maps for all the SDCs (see Fig. 1).

The column density we measure towards an IRDC is integrated along the line of sight and is the sum of the dust column density from the IRDC and the warmer column density from the background. These two components have potentially different dust properties (temperature and specific opacities) and column densities. In some places, the background column density can be higher than the column density of the cloud itself. It is possible to reconstruct the background first and remove its contribution to the observed fluxed towards the IRDCs (Peretto et al. 2010; Battersby et al. 2011). However, this is a difficult task for such a large sample of objects. For this reason, we decided to use a more practical method that filters out large scale structures in the column density map constructed as described above. For this purpose we used a $10^{\prime}$ wide median filter on the Herschel column density maps to create a background image, and subtracted this median component from the original column density image to

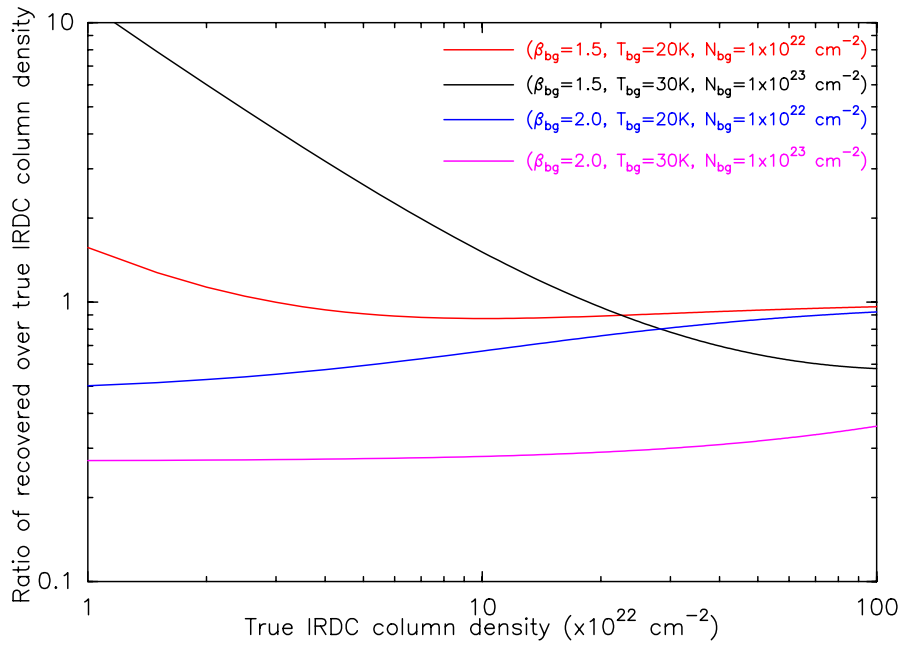

Fig. 3. Uncertainty linked to our column density construction method. In this plot we show the input IRDC column density versus the ratio of the retrieved column density versus true IRDC column density. The different colours correspond to different background properties.

create a background-subtracted column density map (see Fig. 1). These are the maps that we used for the rest of the analysis. The width of the filter was chosen so that it has a similar size to the largest SDCs in the catalogue.

\subsection{The impact of background and SDC dust emission mixing on retrieved cloud properties}

Estimating the column density without separating the background and IRDC contributions to the flux densities could lead to errors on the retrieved column density and temperature of IRDCs. To quantify this error, we modelled the emission of a background and IRDC components as modified-blackbodies at different temperatures and column densities, and added their respective flux densities at both $160 \mu \mathrm{m}$ and $250 \mu \mathrm{m}$. We then used the same procedure as outlined in the previous section to estimate the temperature and column density of the combined IRDC/background components. We finally removed the original background column density from the combined column density to retrieve the IRDC column density. We varied both the properties (column density and dust emissivity index) of the background and the column density of the IRDC itself (with a constant temperature of $12 \mathrm{~K}$ ). In Fig. 3 we show the ratio of the recovered IRDC column density using this technique over the input IRDC column density for different background/IRDC properties. One can see that the errors on the IRDC column densities can be quite high (up to a factor of 10 for low column density IRDCs) for warm and high-column density backgrounds. However, for more typical background properties, the errors are within a factor of 2 . In all cases, the temperature of the IRDC is overestimated by only a few tenths of a Kelvin in the best cases and up to $10 \mathrm{~K}$ in the most difficult cases (low-column density IRDCs against high-column density and warm background).

\subsection{The relative uncertainty of colour versus SED column density maps}

To test our method for calculating the IRDC column densities further, we compared our $160 \mu \mathrm{m} / 250 \mu \mathrm{m}$ colour column densities to the more standard four points [160, 250, $350,500 \mu \mathrm{m}$ ] SED fitting technique. We computed the column 


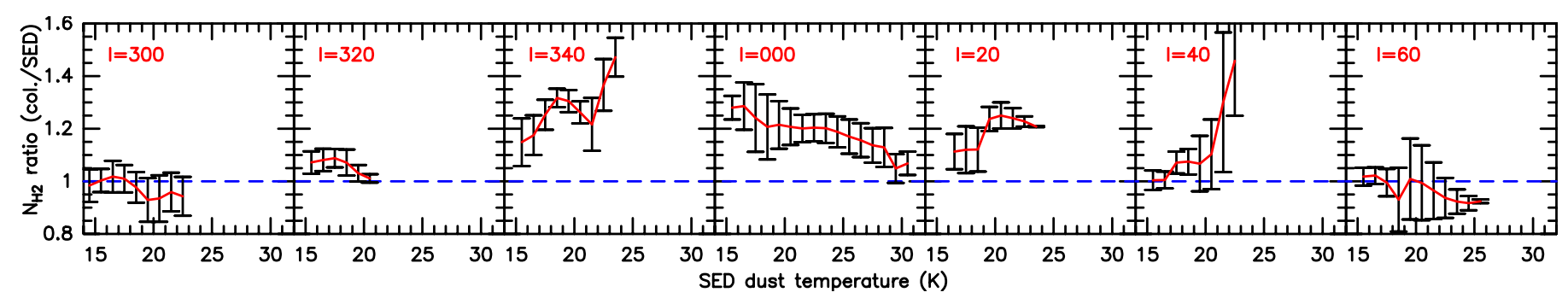

Fig. 4. Ratio of the colour-based over SED column densities as a function of SED dust temperature for entire Hi-GAL tiles. The red solid line shows the average ratio over the corresponding tile, while the error bars represent one $\sigma$ deviations. The blue dashed line indicates a ratio of 1 .

densities following the two methods for entire Hi-GAL tiles at six different locations in the Galaxy and made a pixel-bypixel ratio of the resulting column densities after convolving our colour column density map to the same $36^{\prime \prime}$ resolution of the SED column density map. Figure 4 shows how this ratio varies as a function of dust temperature for all tiles. In this plot, IRDCs correspond to the points at lowest temperatures. We can see that the agreement between the two techniques remains within $\sim 30 \%$ in most cases. The agreement is even better for typical SDC temperatures $(<20 \mathrm{~K})$ and improves when moving away from the Galactic centre. The different trends observed can probably be explained by changes in dust properties, but a full investigation of this is beyond the scope of this paper.

Overall, the method we use to calculate the column density is probably accurate within a factor of 2 for most IRDCs, uncertainties being dominated by the background/IRDC component separation. These uncertainties do not include systematic uncertainties on the dust emissivity, which can account for an extra factor of 2 .

\section{SDC detection in Herschel column density maps}

\subsection{Detection criteria}

To identify which SDCs are detectable in the Herschel column density maps, we first computed a map of column density noise. This is constructed by computing the histogram of the background-subtracted column density pixels in a $10^{\prime}$ box centred on each pixel. Then, in a similar manner to Battersby et al. (2011), we computed the dispersion $\sigma_{j}$ of the backgroundsubtracted Hi-GAL images by mirroring the negative values about the histogram peak, and measured the dispersion on the resulting histogram for pixel $j$ (see Fig. 5). This dispersion is representative of the column density fluctuations of the background on scales lower than $10^{\prime}$.

To decide whether an IRDC is real, we defined three criteria. The first one, $c_{1}$, is the difference between the average Herschel column density within the $\tau_{8} \mu \mathrm{m}$ boundary (as defined in Peretto \& Fuller 2009, see Fig. 1) of the IRDC, $\overline{N_{\mathrm{H}_{2}}^{\text {in }}}$, and the average Herschel column density immediately outside this boundary, $\overline{N_{\mathrm{H}_{2}}^{\text {out }}}$. By immediately outside we mean within the rectangular cutouts that have been defined in Peretto \& Fuller (2009) to extract every SDC. The dimensions of these cutouts are twice the size of the SDC in both $x$ and $y$ directions (i.e. the image axes). If the IRDC is real, then we expect

$c_{1}=\left(\overline{N_{\mathrm{H}_{2}}^{\text {in }}}-\overline{N_{\mathrm{H}_{2}}^{\text {out }}}\right)>0$.

The second parameter, $c_{2}$, is defined as

$c_{2}=\overline{N_{\mathrm{H}_{2}}^{\text {in }}} / \overline{\sigma_{\text {in }}} \geq 3$,

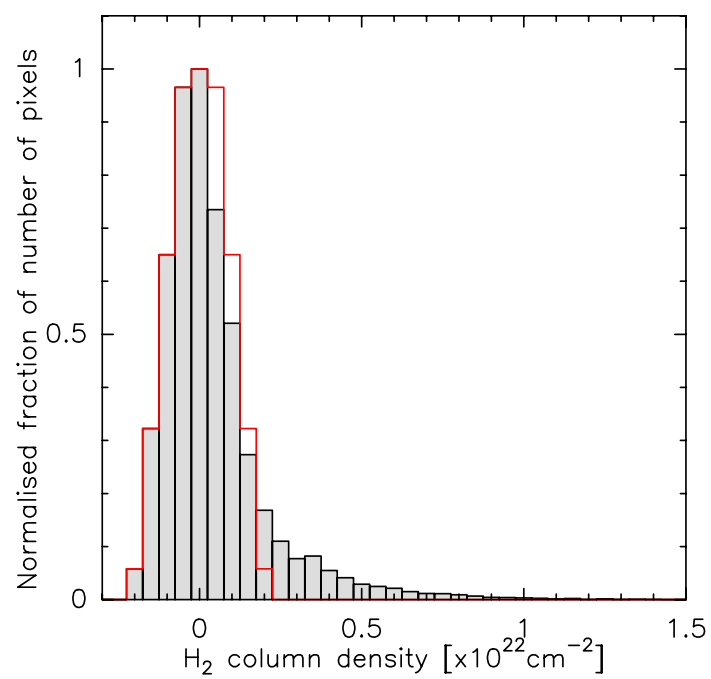

Fig. 5. Histogram of background-subtracted column density pixels (grey histogram) within a $10^{\prime}$ box centred on the central pixel displayed in Fig. 1 . The column density noise estimated in each pixel $\left(\sigma_{j}\right)$ corresponds to the dispersion of the red histogram, obtained by mirroring the negative part of the original histogram about its peak.

where $\overline{\sigma_{\text {in }}}$ is the column density dispersion estimated on the scale of the cloud defined as

$\overline{\sigma_{\text {in }}}=\left\langle\sigma_{j}\right\rangle=\frac{\sum_{j=1}^{n_{\mathrm{pix}}} \sigma_{j}}{n_{\mathrm{pix}}}$ if $R_{\mathrm{eq}}<9^{\prime \prime}$
$\overline{\sigma_{\text {in }}}=\frac{\left\langle\sigma_{j}\right\rangle}{\sqrt{n_{\text {beam }}}}=\frac{\theta_{\text {beam }}}{2 \sqrt{\ln (2)} R_{\mathrm{eq}}} \frac{\sum_{j=1}^{n_{\mathrm{pix}}} \sigma_{j}}{n_{\mathrm{pix}}}$ if $\quad R_{\mathrm{eq}} \geq 9^{\prime \prime}$

where $n_{\text {pix }}$ is the number of pixels within the boundary of the IRDC, $n_{\text {beam }}$ is the number of Herschel beams within the IRDC boundaries, $\theta_{\text {beam }}=18^{\prime \prime}$ the resolution of the Herschel column density maps, and $R_{\text {eq }}$ the equivalent angular radius of the IRDC (as defined in Peretto \& Fuller 2009).

The third criterion, $c_{3}$, is defined as

$c_{3}=\left(\overline{N_{\mathrm{H}_{2}}^{\text {in }}}-\overline{N_{\mathrm{H}_{2}}^{\text {out }}}\right) / \overline{\sigma_{\text {in }}} \geq 3$.

This last criterion is more selective than $c_{2}$, and as a result of eye investigation, we consider that a significant number of real IRDCs would be missed by using it, picking up very high signalto-noise ratio clouds (an example of a SDC meeting $c_{1}$ and $c_{2}$ criteria but failing $c_{3}$ is shown in Appendix A - SDC15.4220.098). The detection results presented in this paper are based on $c_{1}$ and $c_{2}$ alone. 
Table 1. SDC Herschel counterpart properties.

\begin{tabular}{cccccccccc}
\hline \hline Name & $\begin{array}{c}R_{\mathrm{eq}} \\
\prime\end{array}$ & $\begin{array}{c}\overline{N_{\mathrm{H}_{2}}^{\text {in }}} \\
\times 10^{22} \mathrm{~cm}^{-2}\end{array}$ & $\begin{array}{c}\overline{N_{\mathrm{H}_{2}}^{\text {out }}} \\
\times 10^{22} \mathrm{~cm}^{-2}\end{array}$ & $\begin{array}{c}\overline{\sigma_{\mathrm{in}}} \\
\times 10^{22} \mathrm{~cm}^{-2}\end{array}$ & $\begin{array}{c}N_{\mathrm{H}_{2}}^{\mathrm{pk}} \\
\times 10^{22} \mathrm{~cm}^{-2}\end{array}$ & $c_{1}$ & $c_{2}$ & $c_{3}$ & Detected? \\
\hline SDC10.014-0.818 & 65.4 & -0.10 & -0.03 & 0.02 & 0.19 & -0.07 & -4.28 & -2.98 & $\mathrm{n}$ \\
SDC10.031-0.355 & 14.7 & 5.09 & 2.96 & 0.25 & 5.95 & 2.13 & 19.99 & 8.36 & $\mathrm{y}$ \\
SDC10.043-0.425 & 62.6 & 1.19 & 0.71 & 0.06 & 4.69 & 0.48 & 21.30 & 8.62 & $\mathrm{y}$ \\
SDC10.055-0.355 & 20.1 & 1.43 & 0.71 & 0.21 & 2.30 & 0.72 & 6.78 & 3.44 & $\mathrm{y}$ \\
SDC10.067-0.406 & 6.9 & 4.70 & 3.26 & 0.37 & 5.82 & 1.43 & 12.83 & 3.92 & $\mathrm{y}$ \\
SDC10.069-0.400 & 4.2 & 2.83 & 2.59 & 0.38 & 3.65 & 0.24 & 7.44 & 0.64 & $\mathrm{y}$ \\
SDC10.082-0.414 & 25.8 & 2.20 & 1.21 & 0.15 & 3.56 & 0.98 & 14.39 & 6.44 & $\mathrm{y}$ \\
SDC10.086-0.438 & 38.0 & 1.06 & 0.60 & 0.09 & 2.24 & 0.46 & 12.16 & 5.31 & $\mathrm{y}$ \\
SDC10.094-0.415 & 3.8 & 1.49 & 1.48 & 0.35 & 1.62 & 0.01 & 4.21 & 0.02 & $\mathrm{y}$ \\
SDC10.111-0.431 & 12.0 & 0.89 & 1.11 & 0.28 & 1.32 & -0.22 & 3.15 & -0.76 & $\mathrm{n}$ \\
\hline
\end{tabular}

Notes. The full table is available at the CDS.
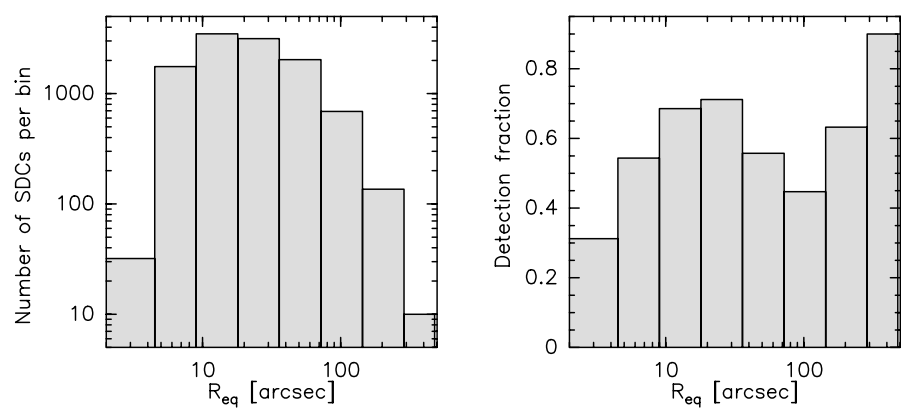

Fig. 6. Number of SDCs (left) and automated detection fraction (right) as a function of SDC angular radius.

For each SDC, Table 1 gives the name, angular radius, $\overline{N_{\mathrm{H}_{2}}^{\text {in }}}$, $\overline{N_{\mathrm{H}_{2}}^{\text {out }}}, \overline{\sigma_{\text {in }}}$, the peak $\mathrm{H}_{2}$ column density estimated with Herschel within the SDC boundary $N_{\mathrm{H}_{2}}^{\mathrm{pk}}, c_{1}$ value, $c_{2}$ value, $c_{3}$ value, and the last column indicates whether the clouds satisfy the $c_{1}$ and $c_{2}$ criteria.

The PF09 catalogue contains 8 SDCs that are not covered by Hi-GAL, and one that is located in a saturated portion of the $250 \mu \mathrm{m}$ Herschel data, leaving a total of 11287 SDCs for analysis. Figure 6 shows the histogram of SDC angular radius ${ }^{1}$, along with the histogram of the fraction of clouds satisfying criteria $c_{1}$ and $c_{2}$ per SDC size bin. For the remainder of this paper, we refer to this fraction as the automated detection fraction.

Using criteria $c_{1}$ and $c_{2}, 63.2 \%(7,139)$ of the 11287 SDCs of the PF09 catalogue are detected with Herschel. One can see in Fig. 6 that this detection fraction varies as a function of radius, with an increase up to an angular radius of $30^{\prime \prime}$, then a decrease down to $R_{\mathrm{eq}} \simeq 100^{\prime \prime}$, and a final increase at larger radius. This detection curve is affected by a number of elements that affect its interpretation. To get more insight into Fig. 6, we decided to simulate the SDC detection process.

\subsection{Detection simulations}

Because of the resolution difference between Herschel at $250 \mu \mathrm{m}$ $\left(18^{\prime \prime}\right)$ and Spitzer at $8 \mu \mathrm{m}\left(\sim 2^{\prime \prime}\right)$, along with the strong fluctuations of the Galactic column density background, some of the

\footnotetext{
1 The radii used here and quoted in Table 1 differ slightly from the ones in PF09. As a result of cloud reprojection a mistake had been made on the size of the pixel of the Spitzer images, which reflected in an overestimate of the SDC radii up to $30 \%$.
}

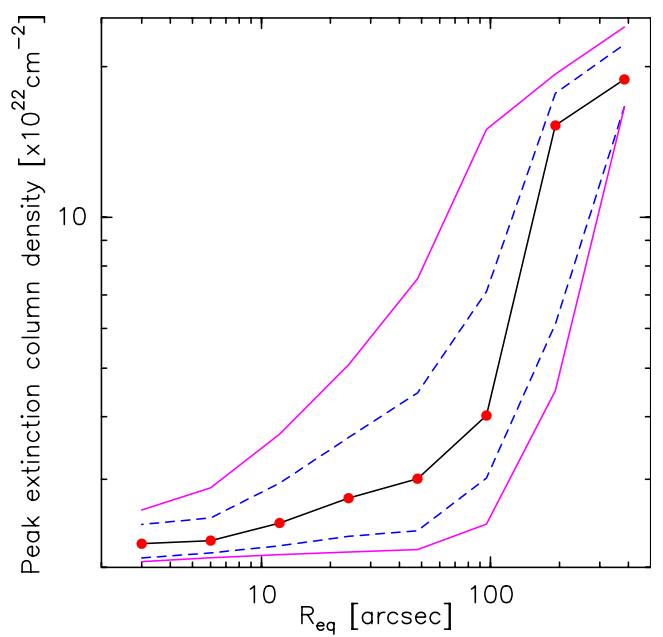

Fig. 7. Angular radius binned the same way as in Fig. 6 versus the median (red symbols and black solid line) peak extinction column densities of all SDCs from the PF09 catalogue. The purple solid lines and blue dashed lines are the 10/90 percentiles and 25/75 percentiles, respectively.

smaller real SDCs may be missed by our identification scheme, while others might be wrongly classified as real. To evaluate the impact of background variations on our detection scheme, and therefore have a better estimate of the fraction of spurious clouds, we performed simulations of our cloud detection method.

Instead of taking idealised cloud models (Bonnor-Ebert spheres for instance), we decided to use SDCs themselves, since we believe they provide a more representative view of detection outcome. It is clear that large clouds with large column density peaks will be more easily detected than small and low-column density ones. To get a sample of SDCs that is representative of the full SDC population, we first computed the angular size versus peak column density (from extinction) for the SDCs from the PF09 catalogue. This is shown in Fig. 7. We can see that the peak column density is smoothly increasing up to $R_{\mathrm{eq}} \simeq 50^{\prime \prime}$ and then increases rather sharply. The last two, and potentially even last three, points of this plot are heavily contaminated by spurious clouds though (cf below). Given this contamination, it seems likely that for real clouds, the trend observed below $R_{\mathrm{eq}}=50^{\prime \prime}$ continues to larger sizes. In any case, as we show below, all real clouds beyond an angular radius of $60^{\prime \prime}$ should 

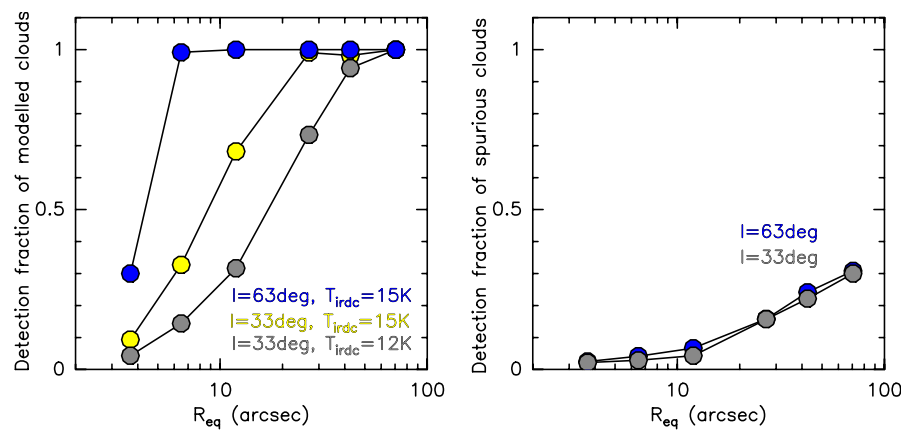

Fig. 8. Results of cloud detection simulations. Left: automated detection fraction of modelled SDCs as a function of SDC size for two different locations in the Galactic plane (i.e. $l \sim 33^{\circ}$ and $l \sim 63^{\circ}$ ) and two different SDC dust temperature (i.e. $12 \mathrm{~K}$ and $15 \mathrm{~K}$ ). Right: automated detection fraction of spurious clouds as a function of size for two different locations.

be detected, no matter what, so for the modelling, we selected a sample of six SDCs whose sizes and column densities follow the median curve (black solid line) of Fig. 7, up to $R_{\text {eq }}=60^{\prime \prime}$.

Using the PF09 Spitzer $\mathrm{H}_{2}$ column density maps, assuming a uniform temperature of either $12 \mathrm{~K}$ or $15 \mathrm{~K}$ (which is representative of the dust temperature of such clouds, Peretto et al. 2010) and the same dust opacity law and molecular weight as in Sect. 2, we inverted Eq. (2) to simulate the appearance of these six clouds at both 160 and $250 \mu \mathrm{m}$. We convolved these images to the Herschel resolutions and placed all six SDCs at 100 different locations within one of the nearly four square degree Hi-GAL tiles of the corresponding wavelength. The longitudes of each location were chosen to be regularly spaced, while the latitudes were randomly drawn from a normal distribution of $F W H M=1^{\circ}$ and central position of $-0.1^{\circ}$, as observed for our IRDC sample (see Fig. 11 of Peretto et al. 2009). We repeated the process for different Hi-GAL tiles between $l=10^{\circ}$ and $l=63^{\circ}$. Finally, we applied our entire identification scheme (i.e. construction of colour column density images and detection criteria) for each modelled cloud. We also calculated $c_{1}$ and $c_{2}$ at the cloud location before adding them to the Herschel images (using the same $\tau_{8 \mu \mathrm{m}}=0.35$ boundaries as for our modelled SDCs - cf. Sect. 4). This allowed us to estimate the probability of having a positive detection even though no SDC is present and therefore gave us a sense of the contamination of the SDC automated detection fraction by spurious features.

Figure 8 displays the main results of our simulations. In the left-hand side panel, we can see that the fraction of modelled clouds that our automated detection scheme manages to identify strongly varies as a function of cloud sizes for all three longitudes displayed here. This fraction reaches 1 if the cloud is larger than $60^{\prime \prime}$ independently of the cloud temperature and location in the Galactic plane. For smaller clouds, the automated detection fraction depends on the cloud temperature and strength of the Galactic background (decreasing from the Galactic centre outwards). Modelled clouds with $R_{\text {eq }} \leq 10^{\prime \prime}$ are the most difficult to identify with an automated detection fraction that could be as low as 0.1 .

On the right-hand side panel of Fig. 8, one can see the fraction of spurious (i.e. non-existent) clouds that manage to pass criteria $c_{1}$ and $c_{2}$ and therefore would be considered as real according to our automated detection scheme. This fraction remains below 0.05 until the cloud reaches an angular radius of $\sim 10^{\prime \prime}$ and then smoothly increases up to $\sim 0.3$ for clouds with $R_{\mathrm{eq}} \simeq 100^{\prime \prime}$. For the largest clouds of the PF09, the automated
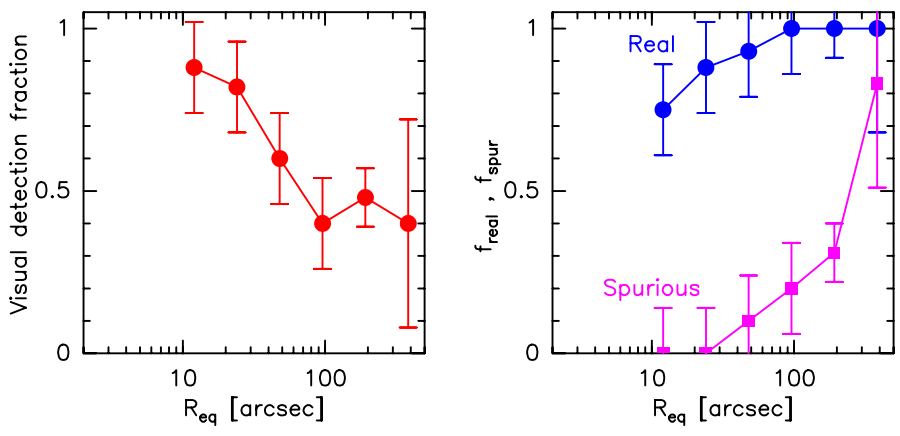

Fig. 9. Left: fraction of real SDCs as estimated through visual inspection (red symbols and solid line). Right: fraction of visually confirmed real SDCs that have been identified as real SDCs by our automated detection scheme ( $f_{\text {real }}$ - blue round symbols), and fraction of visually confirmed spurious SDCs that have been misclassified as real SDCs by our automated detection scheme ( $f_{\text {spur }}-$ purple square symbols). These two curves can directly be compared to our detection simulation results presented in Fig. 8. All error bars correspond to Poisson noise.

detection fraction of spurious clouds can reach 0.5 or more. These spurious detections are related to the probability of getting high column density peaks in a given area; i.e. the larger the area, the higher the probability. It also explains the break in the size column density plot of Fig. 7.

These simulations demonstrate that the automated detection fraction displayed in Fig. 6 is not easily interpreted. In the following section, we use the results of these simulations to further constrain the number of real SDCs in the PF09 catalogue.

\section{Comparison between visual and automated detection fractions}

\subsection{Visual inspection}

Visually inspecting a sub-sample of SDCs is an important step towards validating our detection scheme and simulations. We completed this step by visually matching the morphology of the SDCs as seen in the $8 \mu \mathrm{m}$ Spitzer images with that of their Herschel column density counterparts. This can only be reliably done for rather large SDCs (i.e. at least the size of the Herschel beam). We thus focused on the six largest size bins of Fig. 6 . We decided to check all 146 clouds falling in the last two bins of Fig. 6 (10 clouds in the last bin and 136 clouds in the one before), and 50 SDCs in each of the preceding four size bins. In practice, for each SDC we visually investigated, we overlaid the corresponding Herschel column density contours (starting from $0.1 \times 10^{22} \mathrm{~cm}^{-2}$ and separated by steps of $0.5 \times 10^{22} \mathrm{~cm}^{-2}$ ) on the Spitzer $8 \mu \mathrm{m}$ image, and decided, after eye inspection, whether a column density peak was convincingly matching at least a portion of SDC. A sample of these images is provided in Appendix A. The detection fraction estimated this way is referred to as a visual detection fraction.

The left-hand side panel of Fig. 9 displays the visual detection fraction (column $N_{\text {real }}^{\text {visu }}$ of Table 2) as red symbols. We see that at large radii, the visual detection fraction is lower than the automated detection fraction as estimated for the same cloud sub-sample (column $N_{\text {real }}^{\text {auto }}$ of Table 2). That the fraction of spurious clouds (column $N_{\text {spur }}^{\text {visu }}$ of Table 2) increases with size is a consequence of the construction of the $8 \mu \mathrm{m}$ opacity maps that are built to identify the SDCs (Peretto \& Fuller 2009). One step involves convolving the original Spitzer $8 \mu \mathrm{m}$ images with a 5' Gaussian kernel. This convolution is performed to construct the mid-infrared background image of the region. However, in 
Table 2. SDC visual inspection summary.

\begin{tabular}{cccccccccc}
\hline \hline Size bin & Nb of SDCs & $\begin{array}{c}N_{\text {real }}^{\text {visu }} \\
\%\end{array}$ & $\begin{array}{c}N_{\text {spur }}^{\text {visu }} \\
\%\end{array}$ & $\begin{array}{c}N_{\text {real }}^{\text {auto }} \\
\%\end{array}$ & $\begin{array}{c}N_{\text {spur }}^{\text {auto }} \\
\%\end{array}$ & $\begin{array}{c}n_{\text {real }} \\
\%\end{array}$ & $\begin{array}{c}n_{\text {spur }} \\
\%\end{array}$ & $\begin{array}{c}f_{\text {real }} \\
\%\end{array}$ & $\begin{array}{c}f_{\text {spur }} \\
\%\end{array}$ \\
\hline $8^{\prime \prime}-16^{\prime \prime}$ & 50 & 88 & 12 & 66 & 34 & 22 & 0 & 75 & 0 \\
$16^{\prime \prime}-32^{\prime \prime}$ & 50 & 82 & 18 & 66 & 34 & 10 & 0 & 88 & 0 \\
$32^{\prime \prime}-64^{\prime \prime}$ & 50 & 60 & 40 & 60 & 40 & 4 & 4 & 93 & 10 \\
$64^{\prime \prime}-128^{\prime \prime}$ & 50 & 40 & 60 & 52 & 48 & 0 & 12 & 100 & 20 \\
$128^{\prime \prime}-256^{\prime \prime}$ & 136 & 48 & 52 & 64 & 36 & 0 & 16 & 100 & 31 \\
$256^{\prime \prime}-512^{\prime \prime}$ & 10 & 40 & 60 & 90 & 10 & 0 & 50 & 100 & 83 \\
\hline
\end{tabular}

places where a bright $8 \mu \mathrm{m}$ region is present, this convolution artificially produces significant structures in the mid-infrared background, which translates into large spurious features in the $8 \mu \mathrm{m}$ opacity maps (examples of such spurious clouds can be found in Appendix A, e.g. SDC329.368-0.437). On the other hand, at small radii, the visual detection fraction is greater. This is because the eye can more easily identify low signal-to-noise sources because it recognises matching shapes in Herschel and Spitzer images.

Assuming that the visual inspection provides the true frac-

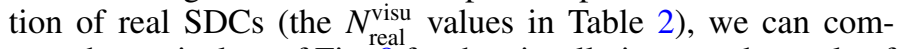
pute the equivalent of Fig. 8 for the visually inspected sample of SDCs. For this, we first need to compute the fraction of SDCs in each size bin that have been misclassified as spurious sources by our automated detection scheme, $n_{\text {real }}$. We also need to evaluate the fraction of SDCs that have been misclassified as real sources by our automated detection scheme, $n_{\text {spur }}$. With these numbers in hand, one can compute $f_{\text {real }}$, the fraction of real SDCs that have been identified as real by our automated detection scheme. This is given by $f_{\text {real }}=\left(N_{\text {real }}^{\text {auto }}-n_{\text {spur }}\right) / N_{\text {real }}^{\text {visu }}$, where $N_{\text {real }}^{\text {visu }}$ is the fraction of visually confirmed SDCs (i.e. the visual detection fraction), and $N_{\text {real }}^{\text {auto }}$ is the automated detection fraction for the same sub-sample of SDCs. This quantity is plotted as blue symbols in the right-hand side panel of Fig. 9, and is directly comparable to the left-hand side panel of Fig. 8. We can also compute $f_{\text {spur, }}$, the fraction of spurious SDCs that have been misclassified as real SDCs by our automated detection scheme. This is given by $f_{\text {spur }}=n_{\text {spur }} / N_{\text {spur }}^{\text {visu }}$, where $N_{\text {spur }}^{\text {visu }}$ is the fraction of visually confirmed spurious SDCs. This quantity is plotted as purple symbols in the right-hand side panel of Fig. 9, and is directly comparable to the right-hand side panel of Fig. 8 (a summary of the visual inspection is given in Table 2). We can see that both trends (the increase in spurious detection fraction with increasing radius and the decrease in real SDC detection fraction with decreasing radius) were predicted by our detection simulations. The amplitude of these two effects are also reproduced well.

Overall, we can reasonably say that the large majority of SDCs with an angular radius under $60^{\prime \prime}$, which have been identified as real by our automated detection scheme, are indeed real. For larger clouds, visual inspection of individual sources is required to check their nature (real versus spurious). We note as well that the extinction-based column density and therefore sizes of the largest clouds appear comparatively uncertain. If any portion of one of these clouds was clearly associated with a Herschel column density peak, we then classified the clouds as real.

\subsection{The fraction of real and spurious SDCs}

The main unknown in determining the overall reliability of the SDCs is the detailed behaviour of the number of real clouds of small sizes that Herschel cannot resolve. For larger sources, a

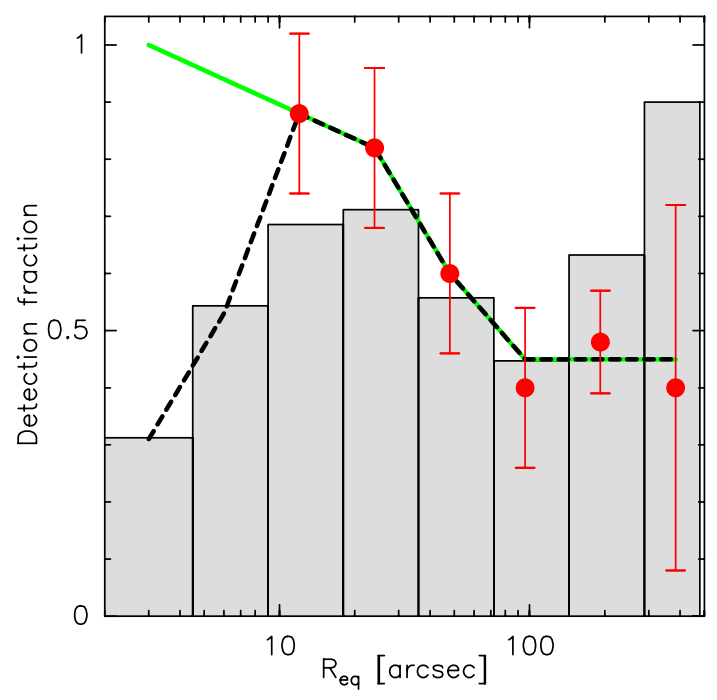

Fig. 10. Detection fraction as a function of SDC angular radius. The grey histogram is the same as in the right-hand side panel of Fig. 6 . The red symbols are the same as in the left-hand side panel of Fig. 9. The black dashed and green solid lines represent the two assumptions that have been made for the fraction of real SDCs (Sect. 5.2).

good estimate of the number of real SDCs as a function of size is provided by the visual detection fraction extrapolated to the entire sample. For the two smallest bins, the real SDC fraction remains unknown. However, given that the trend shows an increase in the number of real SDCs with decreasing sizes (as expected from the simulations), one could argue that the real SDC fraction must keep increasing for the two smallest size bins. The other extreme assumption one can make is that the real SDC fraction at these sizes is given by the automated detection fraction. These two hypotheses are represented in Fig. 10.

Taking the average of these two assumptions, one can now estimate the integrated real fraction of clouds over the entire sample. The percentage of real SDCs is estimated to be $76( \pm 19) \%$; however for clouds with angular radius above $32^{\prime \prime}$, this percentage goes down to $55( \pm 12) \%$. This decrease in the number of real clouds of large sizes is a reflection of the increased presence of artefacts in the $8 \mu \mathrm{m}$ background images (see Sec. 5.1). The quoted uncertainties result from the combination of a $3 \%$ uncertainty related to the two different assumptions regarding the percentage of real small SDCs (see above); a $14 \%$ Poisson uncertainty per size bin related to the small number statistics of the visual inspection, going down to $6 \%$ when considering all six size bins and to $7 \%$ when only considering the four largest size bins; and finally a systematic error of $10 \%$ related to the visual real/spurious classification, which goes down to $5 \%$ when only considering the largest clouds (it is easier to visually characterise the nature of larger clouds). 

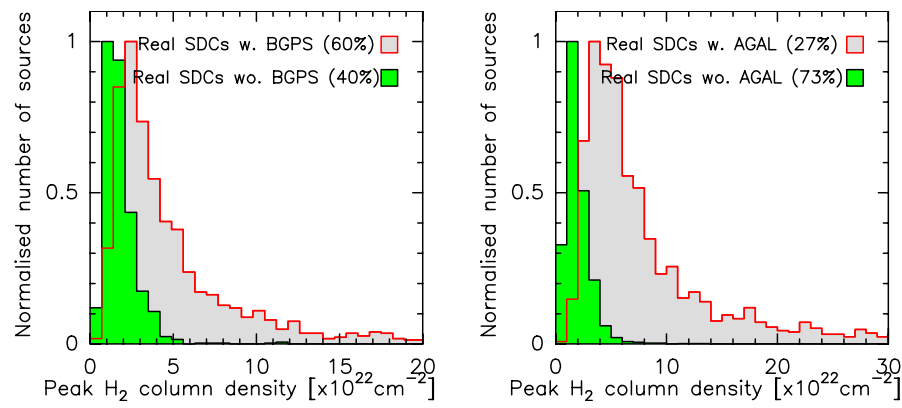

Fig. 11. Left: Herschel $\mathrm{H}_{2}$ peak column density histogram of Herschelconfirmed SDCs with a BGPS counterpart (grey histogram) and without a BGPS counterpart (green histogram). Right: same as the left-hand histogram but for ATLASGAL counterparts. The percentage of Herschelconfirmed SDCs in each category is indicated in the top right corner of each panel.

It is worth noting that using SCUBA $850 \mu \mathrm{m}$ data, Parsons et al. (2009) estimated that $75 \%$ of the 205 MSX IRDCs from the Simon et al. (2006) catalogue they analysed were real. This percentage is in total agreement with the detection fraction we provide here for the Spitzer IRDCs.

For comparison, we checked on a one-to-one basis our detection results with the one from Wilcock et al. (2012) for the $l=\left[300^{\circ}-330^{\circ}\right]$ region. Where they identified $38 \%$ of the SDCs of this region as being Herschel bright, we detect $61 \%$, which is only marginally less than the average over the entire sample. Of these $38 \%$ identified by Wilcock et al. (2012), 82\% are also identified as real by our detection scheme. Of the remaining $18 \%$, $72 \%$ have angular radius smaller than $16^{\prime \prime}$, corresponding to the size bins for which our identification scheme is less complete (see Fig. 10).

The clouds we positively identified but which were missed by Wilcock et al. (2012), i.e. $23 \%$ of the cloud population in the $l=\left[300^{\circ}-330^{\circ}\right]$ region, are mostly low column density clouds that appear to be faint at $250 \mu \mathrm{m}$. This explains why they were missed, based on a visual inspection at that wavelength. An example of such a cloud is given in Fig. 1 .

\section{Spitzer dark clouds in the BGPS and ATLASGAL}

To characterise Spitzer dark clouds further, we cross-checked the Herschel-confirmed SDC sample against the catalogues of both BGPS (Rosolowsky et al. 2010) and ATLASGAL (Csengeri et al. 2014) (sub-)millimetre surveys. The differences in angular resolution, data type (emission versus extinction), and shapes of these sources make the association difficult to define. We considered that there was association between a SDC source and a BGPS/ATLASGAL source when the distance between the centroid positions of the two sources was less than the sum of their radii. The SDC radii are provided in Table 1 of this paper.

For the BGPS sources, we used the values quoted in Col. 10 of Table 1 of Rosolowsky et al. (2010). For ATLASGAL sources, we used, as source radius, the values quoted in Col. 8 of Table 1 of Csengeri et al. (2014). Figure 11 shows histograms of peak $\mathrm{H}_{2}$ column density (see Table 1) for Herschel-confirmed SDCs with and without BGPS counterparts (left panel) and real SDC with and without ATLASGAL counterparts (right panel). In this figure we can see that 1408 of the $2333(60 \%)$ of the real SDCs covered by the BGPS have a BGPS counterparts while $925(40 \%)$ do not. In this histogram it is clear that the latter represent the lowest column density (and smallest) SDCs of the catalogue. These are missed by BGPS as a result of their small sizes and their correspondingly small BGPS beam filling factor. In the right-hand panel of Fig. 11, one can see that only $1907(27 \%)$ of the real SDCs covered by ATLASGAL have an ATLASGAL counterpart. This fraction is in good agreement with Contreras et al. (2013), who find an association fraction of $30 \%$. Here again, SDCs without an ATLASGAL counterpart are mostly at low column density. The reason that the percentage of SDCs with BGPS sources is higher is the difference in the source identification schemes used in BGPS and ATLASGAL. The latter focused on the source identification on rather compact (upper limit of 50") and centrally concentrated (as imposed by the Gaussian fitting routine) sources. Such constraints are not imposed in the BGPS extraction. This comparison shows there is a rather large population of cold and compact sources that are missed by current (sub-)millimetre galactic plane surveys.

We also note that a large number of BGPS and ATLASGAL sources do not have SDC counterparts. While the majority of these sources are infrared bright sources, so cannot be associated, by definition, with an infrared dark cloud, some of them are infrared dark sources that have not been included in the the PF09 catalogue. Ellsworth-Bowers et al. (2013) identify a sub-sample of infrared dark BGPS sources and cross-checked against the SDCs from PF09. In their study, Ellsworth-Bowers et al. (2013) find that $\sim 70 \%$ (based on their Fig. 15) of the sources from their sample are low-contrast infrared dark sources that remained undetected by PF09. In their paper, Ellsworth-Bowers et al. (2013) considered two sources to be associated if the distance between the centroid of the two sources is less than the semi-major axis of the SDC source. This is a very restrictive association condition for two main reasons. The first reason is linked to the definition of the semi-major axis. The semi-major axis $\sigma_{\text {maj }}$ of a SDC is defined as the column density weighed distance dispersion from the centroid position in the direction of the source major axis. Therefore, the disc of area $\pi \sigma_{\text {maj }}^{2}$ will have a much smaller area than $\pi R_{\mathrm{eq}}^{2}$ where $R_{\mathrm{eq}}$ is the radius of the disc of the same area as the source, and BGPS sources outside the disc of radius $\sigma_{\text {maj }}$ will be missed.

The second reason is linked to the shape of SDCs. Sources with elongated or complicated shapes will have a large amount of their area beyond the association radius (even when considering the $R_{\mathrm{eq}}$ as the association radius), and very elongated filaments with BGPS sources at their tips, will be missed. This is exactly what happened for the source that Ellsworth-Bowers et al. (2013) use for illustration in their paper (BGPS \# 5647) and for which they claim that the lower part of the cloud is not part of the PF09 catalogue, while it actually is (seen by looking at the image of SDC35.527-0.269 on wWw. irdarkclouds . org).

We therefore performed the association of IR dark BGPS sources with Herschel-confirmed SDC sources using the same association condition as previously and built the histograms of infrared contrast (see equation 11 of Ellsworth-Bowers et al.

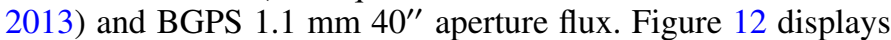
these histograms. First, we see that, with our association condition, only $29 \%$ of BGPS sources are not associated to a SDC as opposed to $70 \%$ in Ellsworth-Bowers et al. (2013) analysis. In the left-hand panel of Fig. 12, we see that this population of sources mostly includes low infrared-contrast sources, as already noted by Ellsworth-Bowers et al. (2013). However, the corresponding distribution is much more peaked (see Fig. 15 of their paper). Looking at images of individual sources with contrast above 0.2 , we also notice that these BGPS sources are, in fact, associated with SDCs. The reason for which we failed to associate these BGPS sources with SDCs is the same as the one mentioned 

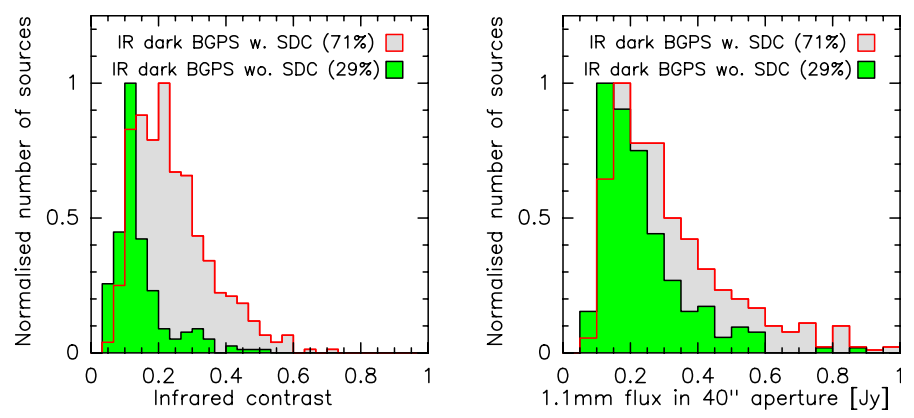

Fig. 12. Left: distribution of infrared contrast for BGPS sources associated with Herschel-confirmed SDCs (grey) and BGPS sources without SDC association (green). Right: same as in the left-hand panel but showing the distribution of BGPS $1.1 \mathrm{~mm}$ flux as estimated within a $40^{\prime \prime}$ aperture radius. The percentage of BGPS sources in each category is indicated in the top right corner of each panel.

above: an elongated BGPS source that includes a SDC at its tip can fail to pass the association criterion because the distance between the centroids of both sources can be larger that the sum of their $R_{\text {eq }}$ radii. These same relatively high contrast sources are also the ones making the high-end tail of the $1.1 \mathrm{~mm}$ flux distribution in the right-hand panel of Fig. 12.

To determine the nature of the remaining low-contrast infrared dark BGPS sources without SDC association, we looked at both their BGPS and $8 \mu \mathrm{m}$ Spitzer images. These sources appear to be mostly low column density IRDCs, as suggested by the position of the peak of $1.1 \mathrm{~mm}$ flux distribution with a large beam filling factor (as opposed to the population of low column density SDCs undetected in BGPS data - see green histogram in Fig. 11). These sources are either isolated sources or they lie in the low-density outskirts of denser clumps.

\section{Summary and conclusion}

Using Herschel Hi-GAL data, we constructed $\mathrm{H}_{2}$ column density images of the Galactic plane at $18^{\prime \prime}$ resolution using the $160 \mu \mathrm{m} / 250 \mu \mathrm{m}$ ratio as a probe of the dust temperature. We used these data to determine the fraction of real IRDCs from the Peretto \& Fuller (2009) catalogue by analysing their Herschel column density properties. Simulating the detection process, along with visually inspecting a small sub-sample of SDCs, shows that small angular size clouds are missed by our automated identification scheme as a result of beam dilution and large background fluctuations. On the other hand, very large features can be wrongly identified as real clouds owing to the probability of finding an Herschel column density peak in a given area of the Galactic plane. Taking these effects into account, we estimated that $76( \pm 19) \%$ of the SDCs are real. This fraction decreases to $\sim 55( \pm 12) \%$ when considering clouds with an angular radius larger than $\sim 30^{\prime \prime}$.
The availability of Herschel data towards the sources of the PF09 catalogue gives us the opportunity to analyse their farinfrared counterparts. As a result, the SDC properties are much better constrained, and studies of the earliest stages of Galactic star formation can now be more reliably performed on both individual and global scales. One particularly interesting feature of this Herschel-confirmed SDC sample is the broad range of cloud sizes and column densities it probes, providing a unique opportunity to study the link between the earliest stages of low- and high-mass star formation across the Milky Way.

Acknowledgements. We thank the anonymous referee whose report helped improve the quality of this paper. N.P. acknowledges the support from the UK Science \& Technology Facility Council (STFC) via grant ST/M000893/1. C.L. has been funded by a STFC Ph.D. studentship. G.A.F. and A.T. acknowledge the support from STFC via grant ST/J001562/1. M.A.T. acknowledges support from STFC via grant ST/M001008/1.

\section{References}

Aguirre, J. E., Ginsburg, A. G., Dunham, M. K., et al. 2011, ApJS, 192, 4 Battersby, C., Bally, J., Ginsburg, A., et al. 2011, A\&A, 535, A128 Bernard, J.-P., Paradis, D., Marshall, D. J., et al. 2010, A\&A, 518, L88 Churchwell, E., Babler, B. L., Meade, M. R., et al. 2009, PASP, 121, 213 Contreras, Y., Schuller, F., Urquhart, J. S., et al. 2013, A\&A, 549, A45 Csengeri, T., Urquhart, J. S., Schuller, F., et al. 2014, A\&A, 565, A75 Ellsworth-Bowers, T. P., Glenn, J., Rosolowsky, E., et al. 2013, ApJ, 770, 39 Griffin, M. J., Abergel, A., Abreu, A., et al. 2010, A\&A, 518, L3 Hildebrand, R. H. 1983, Quant. J. Roy. Astron. Soc., 24, 267 Kauffmann, J., \& Pillai, T. 2010, ApJ, 723, L7

Molinari, S., Swinyard, B., Bally, J., et al. 2010, A\&A, 518, L100 Molinari, S., Schisano, E., Elia, D., et al. 2016, A\&A, in press, DOI: $10.1051 / 0004-6361 / 201526380$

Ossenkopf, V., \& Henning, T. 1994, A\&A, 291, 943

Ott, S. 2010, in Astronomical Data Analysis Software and Systems XIX, eds. Y. Mizumoto, K.-I. Morita, \& M. Ohishi, ASP Conf. Ser., 434, 139

Parsons, H., Thompson, M. A., \& Chrysostomou, A. 2009, MNRAS, 399, 1506

Perault, M., Omont, A., Simon, G., et al. 1996, A\&A, 315, L165

Peretto, N., \& Fuller, G. A. 2009, A\&A, 505, 405

Peretto, N., \& Fuller, G. A. 2010, ApJ, 723, 555

Peretto, N., Fuller, G. A., Plume, R., et al. 2010, A\&A, 518, L98

Peretto, N., Fuller, G. A., Duarte-Cabral, A., et al. 2013, A\&A, 555, A112

Peretto, N., Fuller, G. A., André, P., et al. 2014, A\&A, 561, A83

Piazzo, L., Calzoletti, L., Faustini, F., et al. 2015, MNRAS, 447, 1471

Pilbratt, G. L., Riedinger, J. R., Passvogel, T., et al. 2010, A\&A, 518, L1

Poglitsch, A., Waelkens, C., Geis, N., et al. 2010, A\&A, 518, L2

Ragan, S. E., Bergin, E. A., \& Gutermuth, R. A. 2009, ApJ, 698, 324

Rathborne, J. M., Jackson, J. M., \& Simon, R. 2006, ApJ, 641, 389

Rosolowsky, E., Dunham, M. K., Ginsburg, A., et al. 2010, ApJS, 188, 123

Schuller, F., Menten, K. M., Contreras, Y., et al. 2009, A\&A, 504, 415

Simon, R., Jackson, J. M., Rathborne, J. M., \& Chambers, E. T. 2006, ApJ, 639, 227

Teyssier, D., Hennebelle, P., \& Pérault, M. 2002, A\&A, 382, 624

Traficante, A., Calzoletti, L., Veneziani, M., et al. 2011, MNRAS, 416, 2932

Traficante, A., Fuller, G. A., Peretto, N., Pineda, J. E., \& Molinari, S. 2015, MNRAS, 451, 3089

Wilcock, L. A., Ward-Thompson, D., Kirk, J. M., et al. 2012, MNRAS, 424, 716 


\section{Appendix A: A sample of randomly selected SDCs}
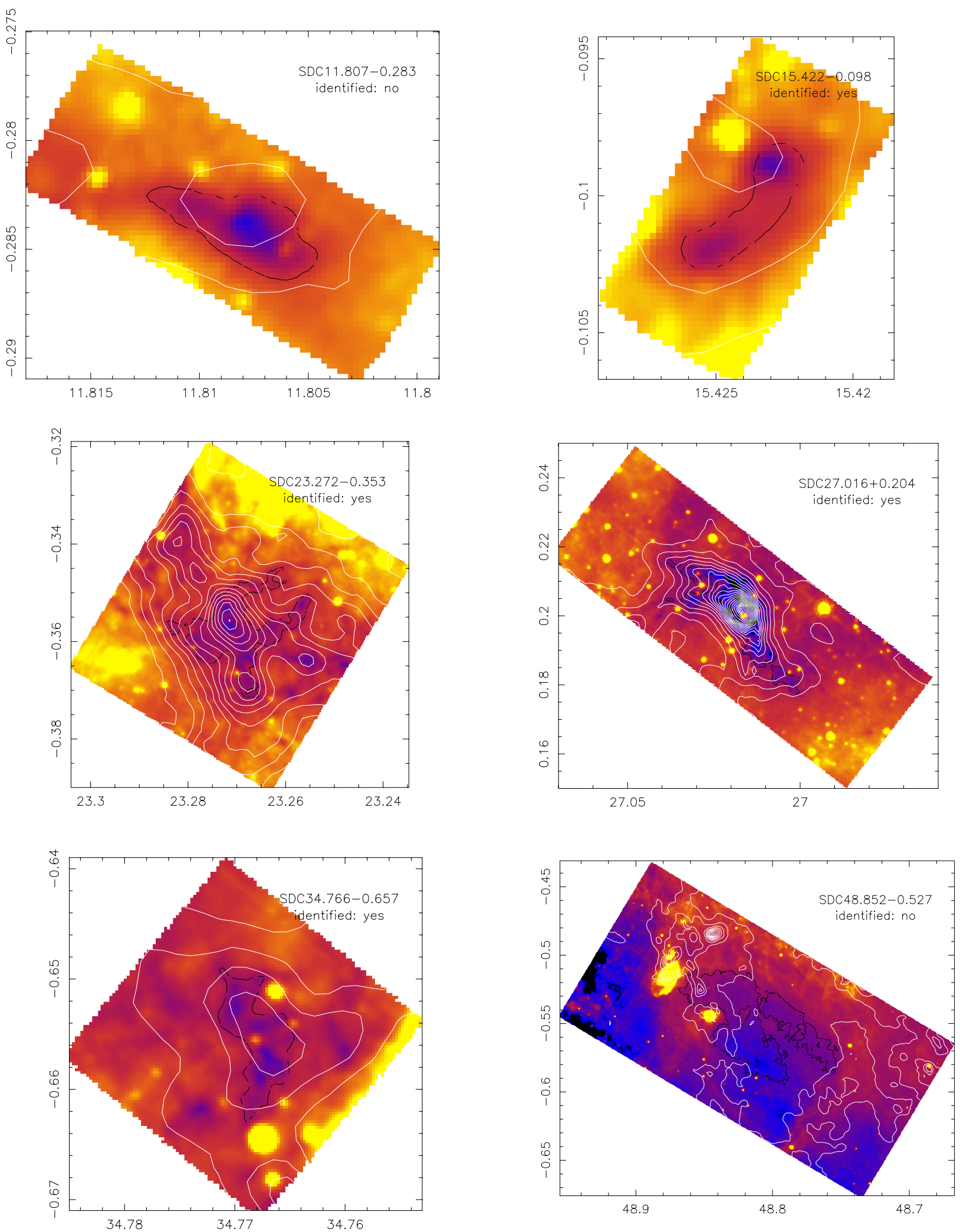

Fig. A.1. Images of 6 randomly selected SDCs. The colour scale is the Spitzer $8 \mu \mathrm{m}$ emission. The black contour is the $\tau_{8} \mu \mathrm{m}=0.35$ contour marking the boundary of the SDC as originally identified in PF09. The white contours are the Herschel $\mathrm{H}_{2}$ column density contours, all starting at $0.1 \times 10^{22} \mathrm{~cm}^{-2}$, and separated by $0.5 \times 10^{22} \mathrm{~cm}^{-2}$. The axes of the images are galactic coordinates in degrees. In the top right corner we give the name of the SDC and if our identification scheme has recognised them as being identified with a Herschel column density peak. SDC11.807-0.283 is not identified while, by eye, it seems clearly associated with a faint peak. Criterion $c_{2}$ for this cloud is slightly below our threshold value of 3 , explaining why it is not picked up by our identification scheme. 
N. Peretto et al.: The Herschel counterparts of Spitzer dark clouds
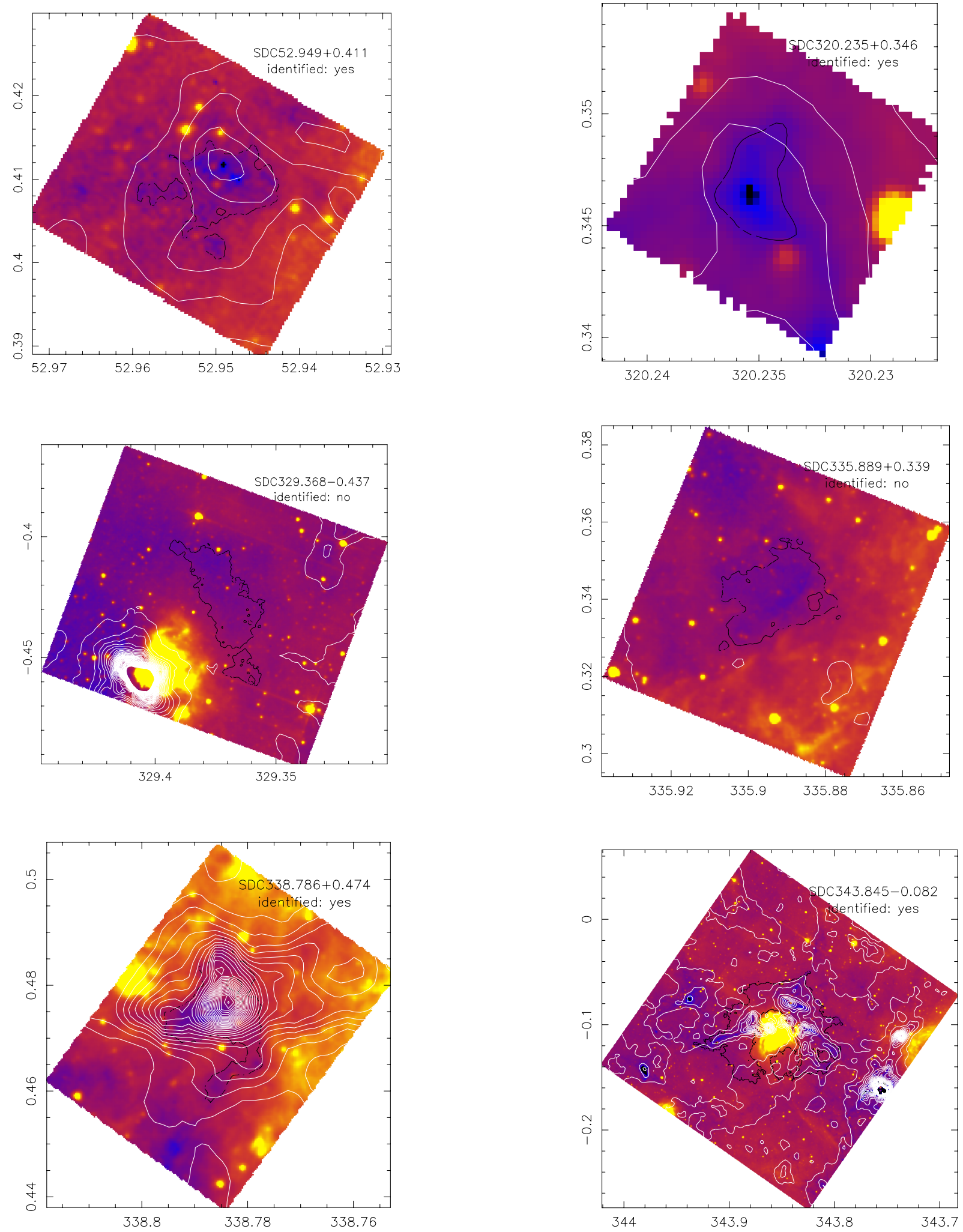

Fig. A.2. Same as Fig. A.1. The Herschel contours for SDC343.845-0.082 have been spaced by $1 \times 10^{22} \mathrm{~cm}^{-2}$ (as opposed to $0.5 \times 10^{22} \mathrm{~cm}^{-2}$ for the others) for clarity. 